Leiriva. $Z_{1}, Z_{2} \in \varphi S \Rightarrow Z_{1} \subset Z_{2} \vee Z_{2} \subset Z_{1}$. contains every element $\nabla$ of $\varphi S$ for which there exists in $\varphi S$ an element $W$. By (3), $Z \in \varphi S$.

The set

$$
\mathscr{S}=\{R \mid(R \epsilon \varphi S \& R \supset Z) \vee R \epsilon \varphi Z\}
$$

is one of the $\mathscr{Z}$ 's in (2) for $S$ as $Z$. For, intersections of sets of elements of $\mathscr{S}$ are again in $\mathscr{S}$ and if $R \epsilon \mathscr{S}$, then $R^{+} \in \mathscr{S}$ (in case $R \supset Z \& R \neq Z$, $\gamma R \notin Z$ since otherwise $R^{+}$would be one of the $V$ 's, with $W=Z$, not contained in $Z$ : a contradiction).

Hence $\varphi S \subset \mathscr{S}$. On the other hand, by $(4), \mathscr{S} \subset \varphi S$, so $\mathscr{S}=\varphi S$. Suppose now $Z \neq \varnothing$. Then $\gamma Z$ must be an element of a set $V$ and $V \neq Z$ by definition of $Z$, so $V \notin Z^{+}$. But by $\varphi S=\mathscr{S}$ we have $V \epsilon \varphi Z$ and (5), (6) imply $V \subset Z^{+}$. Hence $Z=\varnothing$ and the lemma is proved.

We define a mapping $\Phi: S \rightarrow \varphi S$ by

$$
\Phi s=\bigcap_{\substack{Z \in \varphi S \\ s \in Z}} Z \text {. }
$$

Then $s \in \Phi s \neq \varnothing$, and by (1) and (3), $\Phi_{s \in p S .}\left(\Phi_{s}\right)^{+}$is a proper subset of $\Phi_{s}$; so, by $(8), \gamma \Phi s=s$ and $\Phi$ is $1-1$.

Finally we define the relation $\leqslant$ by

$$
s_{1} \leqslant s_{2} \Leftrightarrow \Phi s_{1} \subset \Phi s_{2} .
$$

Using the lemma and the fact that $\Phi$ is $1-1$, we see immediately that $\leqslant$ is a relation of total ordering.

Let $Z$ be a non-void subset of $S$ and let $T$ be the intersection of all elements of $\varphi S$ containing (as subsets of $S$ ) all $\Phi s$ for $s \in Z$. $\gamma T$ must be an element of some $\Phi s_{0}, s_{0} \in Z$. But then $T=\Phi s_{0}$, for otherwise $T$ would be incomparable to $\Phi s_{0}$, contrary to the lemma. Fence $s_{0} \leqslant s$ for all $s \in Z$ and $\leqslant$ is a well-ordering.

\title{
UNIVERSITY OF TOKYO
}

UNIVERSITY OF ZAGREH

Reģu par la Rédaction le 29. 8.1963

\section{REMARKS ON DYADIC SPACES}

BY

\section{R. FNGELKING AND A. PEECZYNSKI (WARSAW)}

Let $D=\{0,1\}$ denote the two point discrete space. For any cardinal number $\mathfrak{m}$ by the $\mathfrak{m}$-Cantor set we mean the Cartesian product $D^{\mathrm{m}}$ of $m$ copies of $D$. The $s_{0}$-Cantor set is a well-known Cantor perfect set on the real line. It is known (see e. g. [9], vol. II, p. 13) that every compact metrizable space is a continuous image of $D^{N_{0}}$. In $[1]$ P.S. Alexandroff defined a dyadic space as a compact space which, for some cardinal number $\mathrm{m}$, is a continuous image of $D^{\mathrm{m}}$, and has raised the problem of whether every compact space is dyadic. This problem was solved in [10] by E. Marezewski, who has shown that every family of non-empty, pairwise disjoint, open sets in $D^{\text {nt }}$ (and then in any dyadic space) is countable, and remarked that the one-point compactifications of high power discrete space are therefore never dyadic (for proofs see [8], p. 166). The class of dyadic spaces was investigated by Sanin [13], Esenin-Volpin [7] and, recently by Efimov [6], [6a].

In this note we give simple proofs of two known theorems ( 1 and 2 ) and we establish two theorems ( 3 and 4 ) which seem to be new. In section 1 theorems 1-4 are formulated and the proofs of theorems 1 and 2 are given. Section 2 contains purely topological proofs of theorems 3 and 4 and two examples in connection with theorem 3 . In section 3 we give proofs of theorems 3 and 4 by using the "function space method", based on the fact that the functor $C(\cdot)$ establishes the contravariant isomorphism of the category of compact spaces with homeomorphic embeddings and continuous mappings onto as morphisms, to the category of Banach algebras of all continuous real-valued functions on compact spaces with homomorphisms onto and isomorphic embeddings as morphisms.

By space we always mean a completely regular space. By $E, I, N$ and $D$, we shall denote the real line, the closed interval $0 \leqslant x \leqslant 1$, the set of positive integers with discrete topology and the two point discrete space, respectively. $D^{\mathfrak{m}}$ and $I^{\mathrm{m}}$ denote the Cartesian product of $\mathfrak{m}$ copies of $D$ and $I$, respectively. The Cech-Stone compactification of a space $X$ is denoted by $\beta X$. It is characterized among the compactifications of $X$ 
(to within a homeomorphism keeping $X$ pointwise fixed) by the fact that every continuous function $f: X \rightarrow I$ has a continuous extension over $\beta X$. This fact implies that every continuous function $f: X \rightarrow Z$ into a compact space $Z$ has a continuous extension over $\beta X$.

1. Let $\left\{X_{s}\right\}_{s \in S}$ be a family of spaces, $\bar{Y}$ a space, and let $f: \underset{s \in S}{\boldsymbol{P}} X_{s} \rightarrow Y$ be a continuous function.

If for some $S_{0} \subset S$ we have $f(x)=f(y)$ for all $\boldsymbol{x}=\left\{x_{s}\right\}$ and $\boldsymbol{y}=\left\{y_{s}\right\}$ in $\underset{s \in S}{\boldsymbol{P}} X_{s}$ such that $x_{s}=y_{s}$ for $s \in \mathcal{S}_{0}$, then we shall say that $f$ depends only on coordinates belonging to $S_{0}$. If $f$ depends only on coordinates belonging to some $S_{0}$ of cardinality at most $m$, then we say that $f$ depends only on $\mathfrak{m}$ coordinates.

We begin with two well-known lemmas (see e.g. [3]):

Lemma 1. Every real-valued function defined on the Cartesian product $\boldsymbol{P} \boldsymbol{X}_{s}$ of compact spaces depends only on $\boldsymbol{\aleph}_{0}$ coordinates.

Proof. Denote by $C$ the family of all real-valued continuous functions on $\underset{s \epsilon S}{\boldsymbol{P}} X_{s}$ depending only on $\boldsymbol{\aleph}_{0}$ coordinates. For very $f \epsilon C$ let $S(f)$ be a countable subset of $S$ such that $f$ depends only on coordinates in $S(f)$. It is easy to see that for every $f, g \in C$ the functions $f+g$ and $f \cdot g$ depend only on coordinates in $S(f) \cup S(g)$ and that any real-valued function $f$ on $\underset{s e S}{\boldsymbol{P}} \boldsymbol{X}_{\boldsymbol{s}}$ which is a uniform limit of the sequence $\left\{f_{i}\right\}$ of functions belonging to $C$ depends only on coordinates in $\bigcup_{i=1}^{\infty} S\left(f_{i}\right)$. Since the family $C$ contains all the constant functions and separates points of $\underset{8 \in S}{\boldsymbol{P}} X_{s}$, the lemma follows from the Stone-Weierstrass Theorem (see e.g. [5], p. 242).

CoRoLlARY. If $X$ is a dyadio space, then, for every real-valued continuous function $g$ on $X$, there exists a compact metrizable subspace $X_{g} \subset \bar{X}$ such that $g(X)=g\left(X_{g}\right)$.

Indeed, the function $f=g h$, where $h$ is a mapping of some $D^{\mathfrak{m}}=$ $=\boldsymbol{P} D_{s}$ onto $X$, depends only on coordinates belonging to some countable $S_{0} \subset S$. Since a continuous image of a compact metrizable space is metrizable, we may take for $X_{g}$ the space $h\left(\underset{s \in S}{\boldsymbol{P}} D_{s}^{*}\right)$, where $D_{s}^{*}=D$ for $s \in S_{0}$ and $D_{s}^{*}=\{0\}$ for $s \in S \backslash S_{0}$.

LEMMA 2. Every function $f$ defined on the Cartesian product $\underset{s \in S}{\boldsymbol{P}} X_{s}$ of compact spaces and with values in a space $\bar{Y}$ of weight $\left(^{1}\right) \mathfrak{m} \geqslant \boldsymbol{x}_{0}$ depends only on $\mathfrak{m}$ coordinates.

(1) The weight of a space $X$ is the minimal eardinality of bases of $X$.
Proof. By Tychonoff embedding theorem, $Y$ ean be regarded as a subspace of $I^{\mathfrak{m}}$ and $f$ as a family of $\mathfrak{m}$ real-valued functions. Since each of these functions depends only on $\boldsymbol{x}_{0}$ coordinates, the function $f$ depends on $\mathfrak{m} \cdot \mathfrak{s}_{0}=\mathfrak{m}$ coordinates.

Since the weight of a continuous image $f(X)$ of a compact space $X$ is not greater than the weight of $X$, we have

COROLLARY. If $X$ is a dyadic space then, for every continuous mapping $g: X \rightarrow Y$, into the space $Y$ of weight $\mathfrak{m}$, there exists a compact subspace $X_{g} \subset X$ of weight $\mathfrak{m}$ such that $g(X)=g\left(X_{g}\right)$.

THEOREM 1 (Šanin [13]). If $X$ is a dyadic space of weight $\mathfrak{m}$, then there exists a continuous mapping of the $\mathfrak{m}$-Cantor set $D^{\mathrm{m}}$ onto $X$.

Proof. By definition there exists, for some cardinal $\mathfrak{n}$, a mapping $g$ of the $\mathfrak{n}$-Cantor set $D^{\mathfrak{n}}=\underset{s \in S}{\boldsymbol{P}} D_{s}$ onto $X$. In virtue of Lemma 2 there is a subset $S_{0} \subset S$ of cardinality at most $\mathfrak{m}$ such that $g$ depends only on coordinates in $S_{0}$. The subspace $D_{*}^{\mathfrak{n}}=\underset{s \in S^{*}}{\boldsymbol{P}} D_{s}^{*} \subset D^{\mathfrak{n}}$, where $D_{s}^{*}=n$ if $s \in S_{0}$ and $D_{s}^{*}=\{0\}$ if $s \in S \backslash S_{0}$, is homeomorphic with the m-Cantor set and it is easy to see that $g_{*}=g \mid D_{*}^{\mathfrak{n t}}$ maps $D_{*}^{\text {nt }}$ onto $X$.

LeMrma 3. For every closed $G_{\delta}$ set $X$ in $D^{\text {nt }}$ there exists a countable set $S_{0} \subset S$ and a closed subset $X_{0} \subset \underset{s \in S_{0}}{\boldsymbol{P}} D_{s}$ such that $X=X_{0} \times \underset{s \omega S \backslash S_{0}}{\boldsymbol{P}} D_{s}$.

Proof. In virtue of a result by Vedenisoff [16] there exists a continuous real-valued function $f: D^{\mathfrak{m}}=\underset{s e S}{\boldsymbol{P}} D_{s} \rightarrow I$ such that $X=f^{-1}(0)$. By Lemma $1, f$ depends only on coordinates in a countable set $S_{0} \subset S$ and $f=f_{1} p$, where $p: D^{\mathfrak{m}}=\boldsymbol{P} D_{s} \rightarrow \boldsymbol{P} D_{s}$ is the projection and $f_{1}: \underset{s \in S_{0}}{\boldsymbol{P}} D_{s} \rightarrow I$. We have then $X=X_{0} \times \underset{s e S \backslash S_{0}}{\boldsymbol{P}} D_{s}$, where $X_{0}=f_{1}^{-1}(0)$.

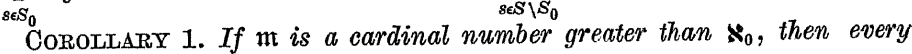
closed $G_{\delta}$ set in $D^{\mathfrak{m}}$ is homeomorphic with $D^{\mathfrak{m}}$.

Indeed, we have shown that $X=X_{0} \times D^{\mathrm{m}}$; by a clasical result (see [9], vol. II, p. 58) $X_{0} \times D^{\mathrm{N} 0}$, as a compact metrizable space not containing isolated points, is homeomorphic with $D^{s_{0}}$. We have then

$$
X=X_{0} \times D^{\mathrm{m}}=X_{0} \times D^{\mathrm{N}_{0}} \times D^{\mathrm{m}}=D^{\mathrm{N}_{0}} \times D^{\mathfrak{m}}=D^{\mathrm{m}}
$$

COROLLARY 2. For every $X$ which is a closed $G_{\delta}$ set in $D^{\mathrm{m}}$ there exists a retraction of $D^{\mathrm{mt}}$ onto $X$.

Indeed, we have shown that $X=X_{0} \times \underset{s \in S \backslash S_{0}}{\boldsymbol{P}} D_{s}$, where $X_{0}$ is a elosed subset of $\aleph_{0}$-Cantor set $\underset{s \in S_{0}}{\boldsymbol{P}} D_{s}$, and hence (see [9], vol. I, p. 169) a retract of $\boldsymbol{P} D_{s}$. This means that there exists a mapping $r: \underset{s \in S_{0}}{\boldsymbol{P}} D_{s} \rightarrow X_{0}$ such that $r(x)=x$ for $x \in X_{0}$. It is easy to see that $X$ is a continuous image of 
$D^{\mathrm{m}}$ by the retraction $g: D^{\mathrm{m}}=\underset{s \in S_{0}}{\boldsymbol{P}} D_{s} \times \underset{s \in S^{\prime} \backslash S_{0}}{\boldsymbol{P}} D_{s} \rightarrow X_{0} \times \underset{s \in S \backslash S_{0}}{\boldsymbol{P}} D_{s}=X$, where $g(x, y)=(r(x), y)$ for every $x \in \underset{s \in S_{0}}{\boldsymbol{P}} D_{s}$ and $y \in \underset{s \in S \backslash S_{0}}{\boldsymbol{P}} D_{s}$.

From Corollary 2 follows

THEOREM 2 (B. Efimov [6]). Every space $X$ which eam be embedded as a closed $G_{\delta}$ in a dyadic space is itself a dyadic space.

In sections 2 and 3 we shall prove the following theorems $\left({ }^{2}\right)$ :

THEOREM 3. If the Cech-Stone compactification $\beta X$ of a space $X$ is dyadic, then $X$ is pseudocompact $\left.{ }^{3}\right)$.

THEOREM 4 . There is no infinite extremally disconnected $\left.{ }^{4}\right)$ dyadio compact space $\left(^{5}\right)$.

2. The following lemma shows that to prove Theorem 3 it is sufficient to show that $\beta E$ is not dyadic. Indeed, if the space $X$ can be continuously mapped onto a dense subset of $Y$ then $\beta Y$ is an image of $\beta X$.

LEMMA 4. For every non-pseudocompact space $X$ there exists a continuous function $f: X \rightarrow E$ such that $f(X)$ is a dense subset of $D$.

Proof. We may confine our attention to $X$ which are non-bounded subsets of $E$. In this case $X$ contains an infinite closed subset $X_{0}=\left\{x_{1}\right.$, $\left.x_{2}, \ldots\right\}$ homeomorphic with a countable discret space. Let $\left\{w_{1}, w_{2}, \ldots\right\}$ be the sequence of all rational numbers. By Tietze extension theorem the function $f_{0}: X_{0} \rightarrow E$ defined by the condition $f_{0}\left(x_{i}\right)=w_{i}$, for $i=1,2, \ldots$, can be extended to a function $f: X-\therefore$. It is easy to see that $f(X)$ is dense in $E$.

Proof of theorem 3. Let $g$ be an extension over $B E$ of the function $f: E \rightarrow I$ defined by

$$
f(x)=\frac{1}{2}\left(\frac{x}{1+|x|}\right)+\frac{1}{2} .
$$
lished)

(2) M. Katětov has remarked that Theorems 3 and 4 follow from his (unpub-

THEOREM. Every non-isolated point of a dyadis space is a limit point of a sequenee of distinct points.

${ }^{(3)}$ By pseudocompact space we mean a space $X$ such that every real-valued function of $X$ is bounded. For normal spaces pseudocompactness coincides with countable compactness.

M. Katětov has first remarked that $\beta N$ is not dyadic. His (unpublished) proof involves properties of the space $A_{7}$, constructed by Alexandroff and Urysohn in [2], which is compact, first countable but non metrizable, and thus, by a theorem due to Esenin-Volpin [7], not dyadic. $A_{7}$ contains a countable dense subset, hence it is a continuous image of $\beta N$, and $\beta N$ cannot be dyadic. We have not succeeded in adapting the elegant reasoning of Katětor so as to get the proof of Theorem 3 ,

(4) A space $X$ is called extremally disconnected if the closure of every open set in $X$ is open; it is easy to see that $X$ is extremally disconnected if and only if any two disjoint open sets in $X$ have disjoint closures.

(5) This theorem has been conjeetured by P. S. Alexandroff.
It is evident that $g(\beta E \backslash E)=\{0,1\}$ and that $g=f$ being one-to-one on $E$ there is no proper compact subspace $X_{g} \subset \beta E$ such that $g\left(X_{g}\right)=g(\beta E)$ $=I$. Since $\beta E$ is not metrizable, we obtain, by Corollary of Lemma 1 , that $\beta E$ is not dyadic.

From Theorem 3 follows

Corollary. The Cech-Stone compactification $\beta X$ of a metric space $X$ is dyadic if and only if $\beta X$ is a metrizable space ( $\left.{ }^{6}\right)$.

Proof of theorem 4 follows, by Theorem 3, from

LEMMA 5. Every infinite extremally disconnected compact space contains, as open subset, a homeomorph of $\beta X_{0}$, for some normal and non-pseudocompact $\bar{X}_{0}$.

Furthernore, $X_{0}$ can be represented as a countable union of pairwise disjoint non-empty closed-open subsets.

Proof. Since $X$ is infinite and extremally disconnected, there exist in $X$ closed-open sets $V_{1}, V_{2}, \ldots$ such that

$$
\nabla_{i} \neq 0 \text { and } \nabla_{i} \cap V_{j}=0 \text { for } i \neq j \text {. }
$$

$X_{0}=\bigcup_{i=1}^{\infty} V_{i}$ is a non-pseudocompact space and, as an $F_{\sigma}$ in normal space $X$, it is normal. Its closure $\bar{X}_{0}$ is a closed-open subset of $X$. To show that $\bar{X}_{0}$ is homeomorphic with $\beta X_{0}$ it is sufficient to prove that every function $f: X_{0} \rightarrow I$ has an extension $f: \bar{X}_{0} \rightarrow I\left({ }^{7}\right)$.

For any disjoint closed sets $A, B \subset I$ the counter-images $f^{-1}(A)$ and $f^{-1}(B)$ are disjoint and closed in $X_{0}$. In view of the normality of $X_{0}$ there exist two open (in $X_{0}$, and thus in $X$ ) sets $U, \nabla$ such that

$$
f^{-1}(A) \subset U, \quad f^{-1}(B) \subset \nabla \quad \text { and } \quad U \frown V=0 .
$$

Since $X$ is extremally disconnected, $\bar{U} \cap \bar{V}=0$ and $\overline{f^{-1}(A)} \cap \overline{f^{-1}(B)}=0$. By a theorem due to Taimanor $[15]$ this fact implies that there exists an extension $\bar{f}$ of $f$ to $\bar{X}_{0} \subset X$.

CoRollary 1. Every infinite extremally disconnected compact space contains a homeomorph of $\beta N$.

Indeed, $X_{0}$ being normal and non-pseudocompact contains a closed subset homeomorphic with $N$. The elosure of $N$ in $\beta X_{0}$ is by Urysohn extension theorem homeomorphic with $\beta N$.

COROLLART 2 (Geba and Semadeni [4]). Every infinite extremally disconnected compact space $X$ can be continuously mapped onto $\beta N$.

Indeed, let $X_{0}=\bigcup_{i=1}^{\infty} \nabla_{i}$, where $\nabla_{i} \neq 0, \nabla_{i} \cap \nabla_{j}=0$ for $i \neq j$ and $\nabla_{i}$ is open in $X_{0}$, be a subset of $X$ such that $\bar{X}_{0}$ is open in $X$ and homeo-

(6) See Appendix at the end of this paper.

(7) This is known, see [5], p. 23, problem $1 \mathrm{H} .6$. We present here another proof. 
morphic with $\beta X_{0}$. The mapping $f: X_{0} \rightarrow N$ such that $f\left(V_{i}\right)=i$ for $i=1,2, \ldots$ can be extended over $\bar{X}_{0}$ and over the whole $X$; every such extension obviously maps $X$ onto $\beta N\left({ }^{8}\right)$.

EXAMTPLE 1. There exists a normal (non-compact) pseudocompact space $X$ such that $\beta X$ is not dyadic.

Let $X$ be a space of all ordinals less than or equal to the first uncountable ordinal $\Omega$ with order topology. It is known (see e.g. [5], p. 74)

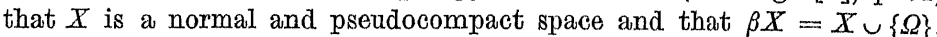
Every non limit ordinal is an isolated point of $\beta X$. It follows then that $\beta X$ contains $\aleph_{1}$ pairwise disjoint non-empty open sets and that $\beta X$ is not dyadic.

EXAMPLE 2. There exists a non-compact space $X$ (pseudocompact by Theorem 3) such that $\beta X$ is dyadic.

Let $X$ be the subspace of $I^{\mathrm{c}}=\underset{s \in S}{\boldsymbol{P}} I_{s}$ composed of such $\left\{x_{s}\right\}$ that $x_{s}=0$ for all but a countable number of $s \in S$. It is shown by Corson in [3] that $I^{c}$ is the Čech-Stone compactification of $X$. Since $I$ is a continuous image of the Cantor perfect set, $I^{\mathfrak{c}}$ is an image of $D^{\mathrm{c}}$, i. $\Theta . \beta X$ is dyadic.

3. If $X$ is a compact space, then $C(X)$ denotes the Banach algebra of all continuous real-valued functions on $X$ with the norm $\|f\|=\sup |f(s)|$. Restating the definition of the dyadic space in the terms of the dual category of algebras of continuous functions we get

Propostrion. A compact space $X$ is dyadic if and only if the algebra $O(X)$ can be isomorphically embedded into an algebra $C\left(D^{\mathrm{m}}\right)$.

This proposition is a consequence of the general fact (see e.g. [5], p. 141) that in order that there exists a continuous mapping from a compact space $X$ into a compact space $Y$ it is necessary and sufficient that the algebra $C(Y)$ can be isomorphically embedded into the algebra $C(X)$.

LEMMA 6. Let $X$ be a dyadic space and let $A$ be a linear olosed subspace of $C(X)$. If $A$ is isomorphic (= linearly homeomorphic) with the Banach space $c$ of all convergent real sequences, then there exists a continuous linear projection from $C(X)$ onto $A$.

Proof. Since $X$ is a dyadic space, $C(X)$ may be considered as a subspace of $C\left(D^{\mathfrak{m}}\right)$ for some $\mathfrak{m} \geqslant \aleph_{0}$. Thus it is sufficient to show that there exists a linear continuous projection $P$ from $C\left(D^{\text {nt }}\right)$ onto $A$; indeed, the restriction of $P$ to $C(X)$ is the required projection.

$\left.{ }^{8}\right)$ R. Sikorski has remarked that by a similar way one can extend Theorem 4 and Corollary 2 to the case of zero-dimensional compact Hausdorff spaces with the property that every open set which is a countable sum of closed-open sets has the open closure.
By the assumption, $A$ is separable, i. e. contains a countable dense subset $f_{1}, f_{2}, \ldots$ Each of the functions $f_{i}$ depends, by Lemma 1 , only on coordinates in some countable set $S_{i} \subset S$. It is easy to see that every function $f \in A$ depends only on coordinates in $S_{0}=\bigcup_{i=1}^{\infty} S_{i}$. Let $B$ be a subalgebra of $C\left(D^{\mathrm{n}}\right)$ consisting of all functions depending only on coordinates in $S_{0}$. Then $B \supset A$ and $B$ is isomorphic, as a ring, to $C\left(D^{N_{0}}\right)$. Since $B$ is separable and $A$ is a Banach space isomorphic with $c$, by a result of Sobczyk [14] (see also [11], p. 217), there exists a linear continuous projection $P_{1}$ from $B$ onto $A$. For any $f \epsilon C\left(D^{\mathrm{m}}\right)$ let $P_{2} f=g$, where $g\left(\left\{x_{s}\right\}\right)=$ $=f\left(\left\{x_{s}^{\prime}\right\}\right)$ and $x_{s}^{\prime}=x_{s}$ for $s \in S_{0}, x_{s}^{\prime}=0$ for $s \notin S_{0}$. It is easily seen that $P_{2}$ is a linear continuous projection from $C\left(D^{\mathrm{m}}\right)$ onto $B$. Finally we put $P=P_{1} P_{2}$.

THEOREM 5. If $X$ is a dyadic space, then no closed linear subspace of $C(X)$ is isomorphic to the space $m$ of all bounded real sequences.

Proof. Suppose, on the contrary, that there is a dyadic space $X$ such that there exists a subspace $B$ of $O(X)$ isomorphic with $m$. Let $A$ be a subspace of $B$ isomorphic with $c$ (for example) the subspace corresponding to the set of all convergent sequences in $m$. Then, by Lemma 6 , there exists a continuous linear projection $P$ from $C(X)$ onto $A$. Thus the restriction of $P$ to the space $B$ would be a linear continuous projection from $B$ onto $A$. But this contradicts the result of Philips [12] that there is no linear continuous projection from $m$ onto its subspace isomorphic with $c$.

Pro of of theorem 3. Suppose that $X$ is a non pseudocompact space. There exists a continuous real-valued function $f: X \rightarrow E$ such that $A=f(X)$ is not bounded. The space $A$ contains, as a closed subset, a space $M$ homeomorphic with $N$. Let $B$ be a subspace of the space $C^{*}(A)$ (of all bounded real-ralued functions on $A$ ) composed of all broken lines with vertices in $M$. It is easily seen that $B$ is a linear subspace of $C^{*}(A)$ isomorphic with $m$. But $C^{*}(A)$ is a linear subspace of $C^{*}(X)=C(\beta X)$ and, by Theorem $5, X$ is not dyadic.

Proof of theorem 4. By a result of Geba and Semadeni [4] (which is the dual restatement of Corollary 2 to our Lemma 5) if $X$ is an extremally disconnected infinite compact space, then the space $O(X)$ contains a subspace isomorphic with $m$. Hence, by Theorem $5, X$ is not dyadic.

Appendix. B. Efimov has kindly communicated to us that the following theorem (which presents a generalization of our Corollary of Theorem 3) is a consequence of his recent results [6a]:

THEOREM. A compactification $r X$ of a metric space $X$ (i. e. a compact space containing $X$ as a dense subspace) is dyadic if and only if $r \bar{X}$ is a metrizable spase. 
We present here another proof of this theorem.

LENaca. For every compactification $r X$ of a separable metric space $X$ there exists a metrizable compactification $r^{\prime} X$ of the space $X$, smaller than $r X$, i. e. such that for some $g: r X \rightarrow r^{\prime} X$ we have $g(x)=x$ for every $x \in X$.

Proof. Let $\exists$ be a countable basis of $X$ and let $P$ be the set of all pairs $(U, W)$ of elements of $\mathfrak{Z}$ such that $\bar{U} \cap \overline{(r X \backslash W)}=0$ (closure in $r X$ ). For any pair $p=(U, W) \in \mathfrak{P}$ let $f_{p}: r X \rightarrow I$ be such that $f_{p}(U)=\{1\}$ and $f_{p}(r X \backslash W)=\{0\}$. It is easy to see that $g=\left\{f_{p}\right\}_{p \in \mathbb{B}}: r X^{r} \rightarrow I^{*_{0}}$ is the homeomorphic embedding and that $r^{\prime} X=g(r X)$ is the required compactification.

Proof of the theorem. It is sufficient to show that if $r X$ is dyadie then $r X$ is metrizable.

It is well known that every non separable metric space contains, for some $\varepsilon>0$, an uncountable family of disjoint $\varepsilon$-spheres. Since in $r X$ every family of open, pairwise disjoint, non-empty sets is countable $X$ is separable. Let $r^{\prime} X$ and $g: r X \rightarrow r^{\prime} X$ be as in Lemma. Because $g(r X \backslash X)=r^{\prime} X \backslash X$ (see [5], p. 92) if $g\left(X_{g}\right)=g(r X)$ for some $X_{g} \subset r X$ then $X \subset X_{g}$. It follows then, by Corollary of Lemma 2 , that $r X$ is metrizable.

Let us note that by the same method one can prove that the weight of any dyadic eompactification $r X$ of a space $X$ is equal to the weight of $X$.

COROLLARX. Every dispersed (not containing perfect subspaces) dyadio space $X$ is metrizable.

Indeed, such $X$ is a compactification of the (discrete) set of its isolated points.

\section{REFERENCES}

[1] П. С. Александров, $K$ теории тополоаииески простраиств, Доканы Академши Наук СCCP 2 (1936), p. $51-54$

[2] P. S. Alexandroff et P. S. Urysohn, Mémoire sur les espaces Lopologiques compacts, Verhandelingen, Akademie van Wetenschappen Amsterdam, I Sectie, XIV (1929), No 1, p. 1-96.

[3] H. H. Corson, Normality in subsets of produet spaces, American Jommal of Mathematics 81 (1959), p. 785-796.

[4] K. Gęba and $\mathrm{Z}$. Semadeni, On the M-subspace of the Banach spaces of continuous functions, Zeszyty Naukowe Uniwersytetu im. Adama Mickiewioza w Poznaniu 2 (1960), p. 53-68.

[5] L. Gillman and M. Jerison, Rings of continuous functions, New York 1960

[6] Б. Ефимов, O диадических бикомпактах, Доклады Академит Науг CCCP 149 (1963), p. 1011-1014.

[6a] - O диадических пространстваx, ibidem 151 (1963), p. 1021-1024.

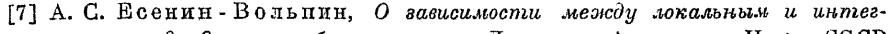
ральныц, весом в $в$ диа 68 (1949), p. $441-444$

[8] J. L. Kelley, General Topology, New York 1955.

[9] C. Kuratowski, Topologie $I, I I$, Warszawa 1958 and 1961.

[10] E. Marczewski (E. Szpilrajn), Remarque sur les produits cartesiens d'espaces topologiques, Доклады Академии Наук CСCP 31 (1941), p. 525-528.

[11] A. Pełczyúski, Projections in certain Banach spaces, Studia Mathematica $19(1960)$, p. $209-228$

[12] R. S. Philips, On linear transformations, Transactions of the Ameri(an Mathematical Society 48 (1940), p. 516-541.

[13] Н. ШІанин, О произведении топологических пространств, Труды Математического Института пм. Стеклова 24 (1948).

14] A. Sobczyk, Projection of the space $(m)$ on its subspace $\left(c_{0}\right)$, Bulletin of the

[15] А. Д. Тайманов, О распостранении непрерывных отображсений топомогических пространсть, Математический Сборник 31 (1952), p. 459-463.

[16] N. Vedenisoff, Sur les fonctions continues dans les espaces topologiques, Fundamenta Mathematicae 27 (1936), p. 234-238.

MATHEMATIOAL INSTITUTE OF THE POLISH ACADEMT OF SCIENCES

Recu par la Rédaction le 12.2.1963 